\title{
Geographic distribution of need and access to health care in rural population: an ecological study in Iran
}

\author{
Aliasghar Ahmad Kiadaliri ${ }^{1,2,3^{*}}$, Behzad Najafi, ${ }^{4,5}$ and Hassan Haghparast-Bidgoli ${ }^{6}$
}

\begin{abstract}
Introduction: Equity in access to and utilization of health services is a common goal of policy-makers in most countries. The current study aimed to evaluate the distribution of need and access to health care services among Iran's rural population between 2006 and 2009.

Methods: Census data on population's characteristics in each province were obtained from the Statistical Centre of Iran and National Organization for civil registration. Data about the Rural Health Houses (RHHs) were obtained from the Ministry of Health. The Health Houses-to-rural population ratio (RHP), crude birth rate (CBR) and crude mortality rate (CMR) in rural population were calculated in order to compare their distribution among the provinces. Lorenz curves of RHHs, CMR and CBR were plotted and their decile ratio, Gini Index and Index of Dissimilarity were calculated. Moreover, Spearman rank-order correlation was used to examine the relation between RHHs and CMR and CBR.

Results: There were substantial differences in RHHs, CMR and CBR across the provinces. CMR and CBR experienced changes toward more equal distributions between 2006 and 2009, while inverse trend was seen for RHHs.

Excluding three provinces with markedly changes in data between 2006 and 2009 as outliers, did not change observed trends. Moreover; there was a significant positive relationship between CMR and RHP in 2009 and a significant negative association between CBR and RHP in 2006 and 2009. When three provinces with outliers were excluded, these significant associations were disappeared.
\end{abstract}

Conclusion: Results showed that there were significant variations in the distribution of RHHs, CMR and CBR across the country. Moreover, the distribution of RHHs did not reflect the needs for health care in terms of CMR and CBR in the study period.

Keywords: Equality, Gini Index, Index of Dissimilarity, Rural Health Houses, Iran

\section{Introduction}

Following Alma-Ata declaration on the key role of primary health care (PHC) in achieving health for all and decreasing inequality in health [1], the Iranian government attempted to develop an extensive network of PHC facilities, especially in rural areas. PHC in Iran's rural areas are mainly provided through the rural health houses (RHHs). RHHs, which are considered as the main component of progressive expansion of PHC coverage, are aimed at reducing the urban-rural gap in Iran's health care delivery

\footnotetext{
* Correspondence: aliasghar.ahmad_kiadaliri@med.lu.se

'Division of Health Economics, Department of Clinical Sciences, Malmö; Skåne University Hospital; Lund University, MALMÖ SE-20502, Sweden Full list of author information is available at the end of the article
}

system [2]. Following a series of pilot projects in early of 1970s, RHHs were introduced in 1981 [3].

RHHs act as the first level of contact to the basic PHC in Iran's rural areas. These units serve a population of 1,500 people who are living in the main village (where $\mathrm{RHH}$ is located), and satellite villages (which are an hour walk distance from the main village). Two trained local residents, who are known as Behvarz (one male and one female), work as health workers in each RHH and provide PHC services including maternal and child health care, family planning, vaccinations and environmental health promotion to the rural population. Moreover, they are responsible for referring patients who need further

\section{Biomed Central}


care to the next level of care. Figure 1 shows the position of RHHs in the health care delivery system of Iran.

During past decades, the number of rural health houses has continuously increased in the country. However, it is not just the quantity of health care resources which affect health status of people, but how they are distributed is also important. Inequitable distribution of health services is a major barrier for improving health service delivery for health systems around the world [4]. There is indeed a positive linkage between availability of health care resources and health status of population [5]. For this reason, the distribution of health care is considered as one of the social determinants of health [6].

In a sense, the geographic distribution of health facilities is considered as a major health policy issue in many countries, both developed and developing. It is believed that the utilization of, and access to, healthcare among individuals should not be affected by the geographical region in which they reside [7].

Although studies in Iran have examined the effectiveness of rural health houses in improvement of the population's health status [8] and decreasing the disparities between rural and urban areas [9], little attention has been paid to the distribution of RHHs within rural areas in the country.

The current study examines availability of RHHs in Iran using inequality measures. This study specifically focuses on the following research questions: How were RHHs, crude mortality rate (CMR) and crude birth rate (CBR) distributed between different provinces in years 2006 and 2009 ? How has changed the inequality measures between 2006 and 2009? Did the distribution of RHHs reflect CMR and $\mathrm{CBR}$ in the rural population of the provinces?

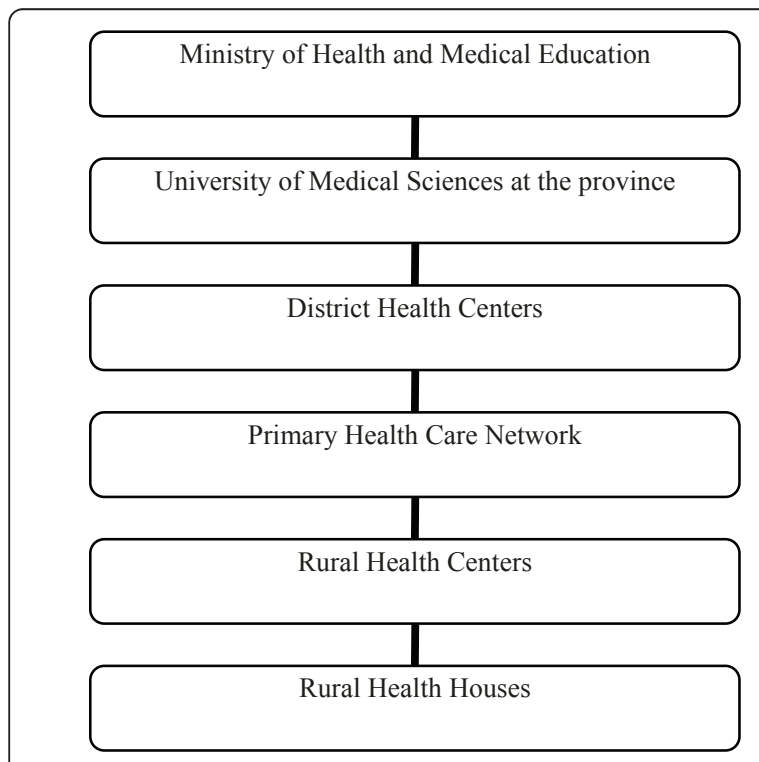

Figure 1 Position of Rural Health Houses (RHHs) in the health care delivery system of Iran.

\section{Material and methods Study setting}

Iran, a lower-middle-income country, is located in Eastern Mediterranean Region with an area of 1,648,000 km sq. It has a population of about 73.5 million people; of whom about 23 million are living in rural areas [10]. The country has 30 provinces, 293 districts, 885 cities and more than 68,000 villages [11]. Table 1 shows some of the main health indicators for the country [10].

\section{Data sources and variables}

The census and estimated data on the distribution and characteristics of population at province level were obtained from the Statistical Centre of Iran [12]. The data about the number of total births and mortalities were also obtained from National Organization for Civil Registration [13]. The data on the number of RHHs in the provinces were gathered from the Statistics Centre of Ministry of Health and Medical Education (MOHME). The centre collects data about health facilities and other health indicators from the Medical Universities in the provinces.

In the current study, the number of RHHs per 1000 rural people (RHP) was used as the indicator for availability of health care resources for the rural population in each province. Moreover, two variables including CMR (number of deaths per 1000 rural people) and CBR (number of births per 1000 rural people) were selected to show the community's health needs. CMR have been used in the literature as an estimate of community health need [14-16]. In addition, CBR was used as another community health need indicator in this study since health services for newborns and infants are one of the main components of the services provided by $\mathrm{RHH}$.

To see how the distribution of access and need to health care services has changed over time, the data for years 2006 and 2009 (as latest available data) were gathered. Moreover, this enables us to control for potential measurement bias in the data as each year can used as a control for other year.

\section{Inequality Indicators}

\section{Lorenz Curve and Gini index}

The Gini index and Lorenz curve are commonly used in analyzing the inequality in distribution of health care resources [17-19]. Lorenz curve is used to compare distribution of specific health variable with perfect equality (diagonal line). This curve plots the cumulative share of population ranked by health variable, in an increasing order, against the cumulative share of health variable. The further the distance from diagonal line, the greater the degree of inequality. The area between the Lorenz curve and diagonal line present a measure of inequality entitled Gini Index. The Gini index is equal to twice the area between the Lorenz curve and diagonal. The 
Table 1 Some main health indicators in Iran

\begin{tabular}{|c|c|c|}
\hline Indicator & Value & Year $^{a}$ \\
\hline Crude birth rate (per 1000 people) & 18.3 & 2006 \\
\hline Crude mortality rate (per 1000 people) & 5.72 & 2009 \\
\hline Population with access to improved sanitation (\%) & 71 & 2008 \\
\hline Total expenditure on health of $\%$ of GDP & 6.3 & 2008 \\
\hline Out-of-pocket expenditure as \% of total health expenditure & 51.7 & 2008 \\
\hline Physicians per 10,000 population & 8.9 & 2008 \\
\hline primary health care units and centres per 10,000 population & 3.1 & 2006 \\
\hline Population with access to local health services, total (\%) & 99 & 2009 \\
\hline Total life expectancy at birth (years) & 72.1 & 2006 \\
\hline Infant mortality rate (per 1000 live births) & 27 & 2005 \\
\hline Under five mortality rate (per 1000 live births) & 27.7 & 2005 \\
\hline Maternal mortality ratio (per 10000 live births) & 25 & 2005 \\
\hline
\end{tabular}

a Reference year for data provided.

magnitude of the Gini index ranges from 0 (perfect equality) to one (maximum possible inequality). In the current study, the formula proposed by Brown [20] was used for calculating the Gini index as follow:

$$
G=1-\sum_{i=0}^{k-1}\left(Y_{i+1}+Y_{i}\right)\left(X_{i+1}-X_{i}\right)
$$

G: Gini Index

$\mathrm{Y}_{\mathrm{i}}$ : cumulative share of $\mathrm{RHH}$ in the ith province

$\mathrm{X}_{\mathrm{i}}$ : cumulative share of population (ranked by RHP) in the ith province

$\mathrm{k}$ : total number of provinces

\section{Decile ratio}

To calculate the decile ratio, the provinces were ranked by RHP. The top $10 \%$ from the top ratio is then divided by the $10 \%$ of the bottom.

\section{Index of Dissimilarity}

This index estimates the proportion of total health variable which would need to be transferred from provinces with health variable values higher than the country's mean to those which values lower than the country's mean to achieve a situation of perfect equality [21]. It is calculated through the formula:

$$
I D=\frac{1}{2} \sum_{\mathrm{i}=1}^{\mathrm{n}}\left|p_{i p}-\mathrm{p}_{\mathrm{ih}}\right|
$$

$\mathrm{p}_{\mathrm{ip}}$ : ith province's population share

$\mathrm{p}_{\mathrm{ih}}$ : ith province's health variable share

\section{Data analysis}

In the current study, the geographic unit of analysis is 30 provinces in Iran. RHP for each province was calculated as the number of RHHs per 1000 rural people. This ratio was used to rank the provinces in drawing the Lorenz curve and calculating the Gini index and decile ratio for access indicator. In case of need indicators, the CBR and CMR were used to rank the provinces.

Inequality measures (including Lorenz curve, Gini, decile ration and dissimilarity index) were used to assess the level of inequality in the distribution of RHHs, CMR and CBR across the provinces.

The spearman rank-order correlation coefficients between RHP, CMR and CBR were calculated to examine if there is any linear relationship between distribution of RHHs and community health needs. Correlation measures have been used in some other studies to evaluate the linear relationship between two variables in examining the inequality in health $[19,22]$.

To find the potential outliers in data, the box plots of percentage changes in CBR and CMR between 2006 and 2009 were used. The results are reported for total sample and non-outlier sample where outliers were removed from total sample. Moreover, in order to explore changes in CMR and CBR over study period; the median was used due to these outliers in the data.

\section{Results}

Table 2 shows the distribution of RHP, CMR and CBR in the provinces of Iran for years 2006 and 2009. There were substantial differences in all indicators across the country. The national mean value of RHP increased by $7 \%$ from 0.75 in 2006 to 0.80 in 2009 . Of the 30 provinces, Tehran was the province with the lowest RHP in both 2006 and 2009, although its RHP increased from 0.26 in 2006 to 0.31 in 2009. Yazd had the highest RHP in both years, with 1.05 and 1.14 in 2006 and 2009, respectively. The differences between the provinces with the highest and the lowest RHP have reduced (from 4.04-fold in 2006 to 3.70-fold in 2009) over the study period. Except for Fars province, the RHPs increased in 
Table 2 Population ${ }^{a}$, RHP ${ }^{b}, \mathrm{CMR}^{\mathrm{c}}$ and CBR ${ }^{\mathrm{d}}$ in provinces of Iran, 2006 and 2009

\begin{tabular}{|c|c|c|c|c|c|c|c|c|}
\hline \multirow[t]{2}{*}{ Province } & \multicolumn{4}{|c|}{2006} & \multicolumn{4}{|c|}{2009} \\
\hline & Population & RHP & CMR & CBR & Population & RHP & CMR & CBR \\
\hline Ardebil & 512.558 & $1.01(5)^{\mathrm{e}}$ & 5.19 & 16.13 & 473.449 & $1.09(6)$ & 5.81 & 18.16 \\
\hline Bushehr & 308.802 & $0.67(24)$ & 6.83 & 18.85 & 280.982 & $0.77(21)$ & 4.85 & 21.47 \\
\hline Chaharmahal & 415.612 & $0.66(25)$ & 5.70 & 21.98 & 407.188 & $0.74(23)$ & 4.82 & 23.32 \\
\hline East Azarbaijan & 1200.917 & $0.88(10)$ & 5.94 & 18.62 & 1137.522 & $0.94(13)$ & 7.70 & 20.75 \\
\hline Fars & 1683.931 & $0.61(28)$ & 5.93 & 15.75 & 1664.832 & $0.56(29)$ & 5.94 & 16.26 \\
\hline Gilan & 1109.110 & $0.85(14)$ & 6.90 & 10.92 & 1063.204 & $0.91(15)$ & 10.00 & 11.84 \\
\hline Golestan & 821.961 & $0.71(22)$ & 4.91 & 22.70 & 799.663 & $0.75(22)$ & 5.48 & 25.19 \\
\hline Hamedan & 722.496 & 0.79 (18) & 5.93 & 17.76 & 663.813 & $0.86(19)$ & 5.97 & 20.11 \\
\hline Hormozgan & 742.349 & $0.63(27)$ & 28.91 & 23.00 & 773.469 & $0.71(26)$ & 4.16 & 22.55 \\
\hline Ilam & 214.556 & $0.88(12)$ & 5.09 & 18.33 & 205.286 & $0.95(10)$ & 5.05 & 16.81 \\
\hline Isfahan & 760.528 & 0.85 & 6.87 & 12.81 & 634.372 & $0.95(12)$ & 6.05 & 16.31 \\
\hline Kerman & 1099.894 & $0.69(23)$ & 3.23 & 17.94 & 1130.619 & $0.73(25)$ & 3.30 & 19.46 \\
\hline Kermanshah & 624.066 & $1.04(2)$ & 5.36 & 15.91 & 595.599 & $1.12(3)$ & 4.72 & 15.29 \\
\hline Khuzestan & 1401.415 & $0.60(29)$ & 11.11 & 21.60 & 1372.389 & $0.63(28)$ & 3.49 & 21.81 \\
\hline Kohkiluyeh & 332.107 & $0.94(7)$ & 13.54 & 21.35 & 324.817 & $1.00(8)$ & 7.79 & 21.77 \\
\hline Kordestan & 584.337 & 1.03 (3) & 5.16 & 18.25 & 555.509 & $1.11(5)$ & 5.58 & 18.47 \\
\hline Lorestan & 696.377 & $0.88(11)$ & 5.98 & 19.41 & 672.260 & $0.93(14)$ & 4.69 & 21.17 \\
\hline Markazi & 419.184 & $0.98(6)$ & 6.78 & 13.12 & 367.709 & $1.12(4)$ & 9.27 & 14.23 \\
\hline Mazandaran & 1368.289 & $0.87(13)$ & 6.03 & 13.42 & 1327.959 & $0.89(16)$ & 8.90 & 14.34 \\
\hline North Khorasan & 419.114 & $0.80(17)$ & 5.48 & 22.93 & 403.909 & $0.87(17)$ & 6.70 & 23.69 \\
\hline Qazvin & 365.225 & $0.71(21)$ & 5.75 & 16.33 & 332.871 & $0.87(18)$ & 7.52 & 16.07 \\
\hline Qom & 63.643 & $0.93(8)$ & 3.66 & 9.87 & 54.347 & $1.07(7)$ & 4.23 & 12.02 \\
\hline Razavi Khorasan & 1781.179 & $0.71(20)$ & 5.08 & 18.93 & 1711.904 & $0.73(24)$ & 7.07 & 19.78 \\
\hline Semnan & 149.183 & $0.92(9)$ & 5.80 & 13.02 & 141.201 & $0.95(11)$ & 13.05 & 13.41 \\
\hline Sistan \& Baluchestan & 1212.544 & $0.65(26)$ & 8.80 & 34.21 & 1300.819 & $0.65(27)$ & 5.82 & 30.71 \\
\hline South Khorasan & 309.725 & $0.81(16)$ & 7.11 & 19.00 & 297.735 & $0.95(9)$ & 21.27 & 18.21 \\
\hline Tehran & 1161.935 & $0.26(30)$ & 1.74 & 9.57 & 962.542 & $0.31(30)$ & 2.66 & 9.36 \\
\hline West Azarbaijan & 1148.505 & $0.76(19)$ & 4.67 & 23.65 & 1110.783 & $0.85(20)$ & 5.09 & 23.98 \\
\hline Yazd & 201.015 & $1.05(1)$ & 4.53 & 10.03 & 185.569 & $1.14(1)$ & 5.60 & 12.66 \\
\hline Zanjan & 405.261 & $1.01(4)$ & 5.59 & 19.32 & 373.461 & $1.13(2)$ & 7.25 & 19.36 \\
\hline Median & - & 0.83 & 5.78 & 18.29 & - & 0.90 & 5.82 & 18.92 \\
\hline Mean & - & 0.75 & 6.80 & 18.39 & - & 0.80 & 6.21 & 19.27 \\
\hline
\end{tabular}

a In thousands.

b Rural health houses per 1000 rural people.

c Crude Mortality Rate (per 1000 rural people).

d Crude Birth Rate (per 1000 rural people).

e Numbers in parentheses show the provinces ranks.

all provinces between 2006 and 2009. Among the provinces, Qazvin had the highest increase in RHP with $22 \%$ and Sistan \& Baluchestan had the lowest increase with $1 \%$.

The median of CMR increased $0.7 \%$ from 5.78 in 2006 to 5.82 in 2009. Tehran had the lowest CMR in both 2006 and 2009. Hormozgan and South Khorasan were the provinces with the highest CMR in 2006 and 2009 , respectively. There were 16.5 - and 8 -fold differences between provinces with the highest and the lowest CMR in 2006 and 2009, respectively. 20 out of 30 provinces experienced an increase in CMR between 2006 and 2009.
The median of CBR increased $0.4 \%$ over the study period. Tehran had the lowest CBR in both 2006 and 2009. The highest CBR was seen in Sistan \& Baluchestan in the same period. There were 3.6- and 3.3-fold differences between the provinces with the highest and the lowest CBR in 2006 and 2009, respectively. 23 out of 30 provinces experienced an increase in CBR between 2006 and 2009.

As provinces of Hormozgan, Semnan and South Khorasan had the outlier values on variables, we reported the total sample results and results after excluding these provinces separately. As there were no significant changes in general results and interpretation, the results of total sample will be discussed in following sections. 
Overall distribution of access and need indicators across the country in 2006 and 2009 are shown in Figure 2. A comparison between Lorenz curves showed that there are no evident changes in the distribution of $\mathrm{RHH}$ and the CBR between 2006 and 2009 (Figure 2). However, the distribution of CMR approached to the perfect equality line.

Table 3 shows the inequality indicators for $\mathrm{RHH}$, CMR and CBR in 2006 and 2009. Among the variables,
RHHs were most equally distributed and the distribution of CMR was least equal. The Gini coefficient for RHH increased by $6.4 \%$ between 2006 and 2009, while during the same period the Gini for CBR and CMR decreased by $10.8 \%$ and $20.8 \%$, respectively.

Table 4 shows the results of the correlation analyses between the access indicator (RHP) and the need indicators (CMR and CBR) in 2006 (Table 3). There was a

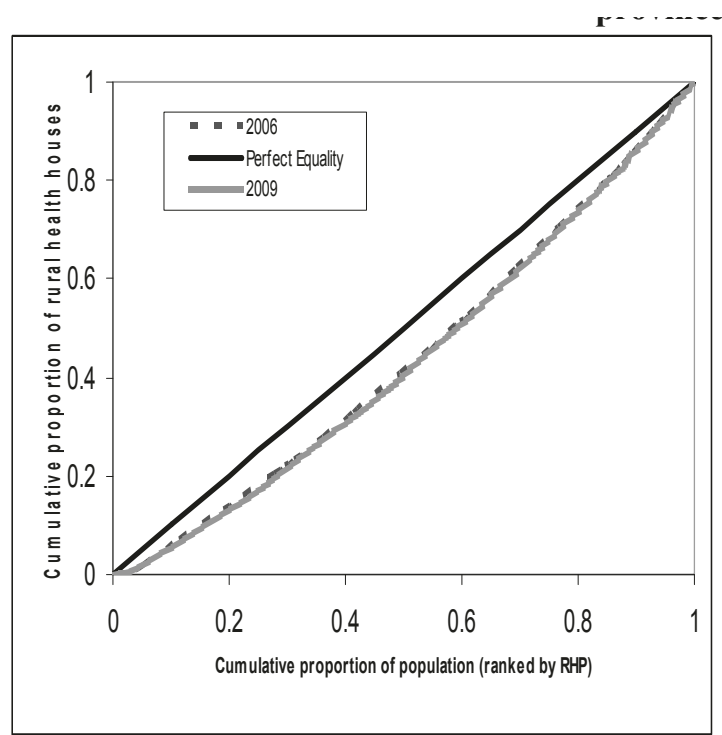

(a)

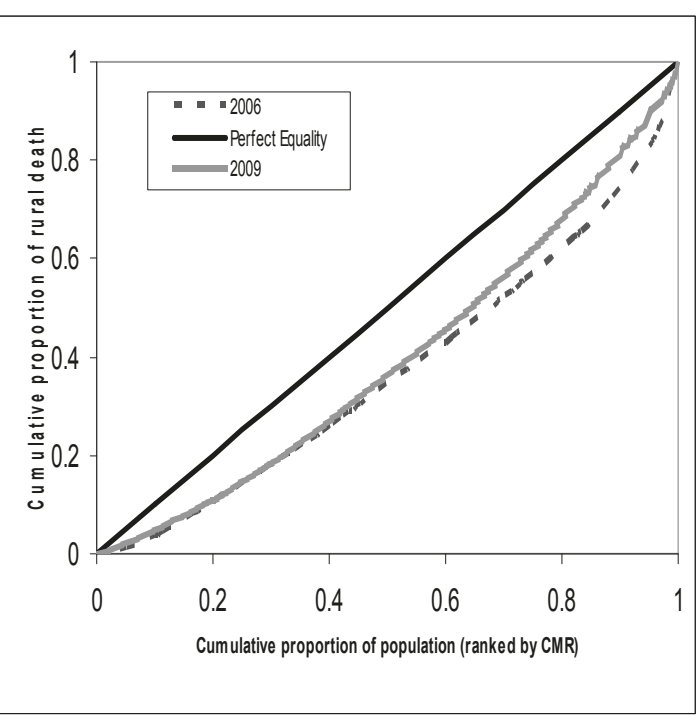

(b)

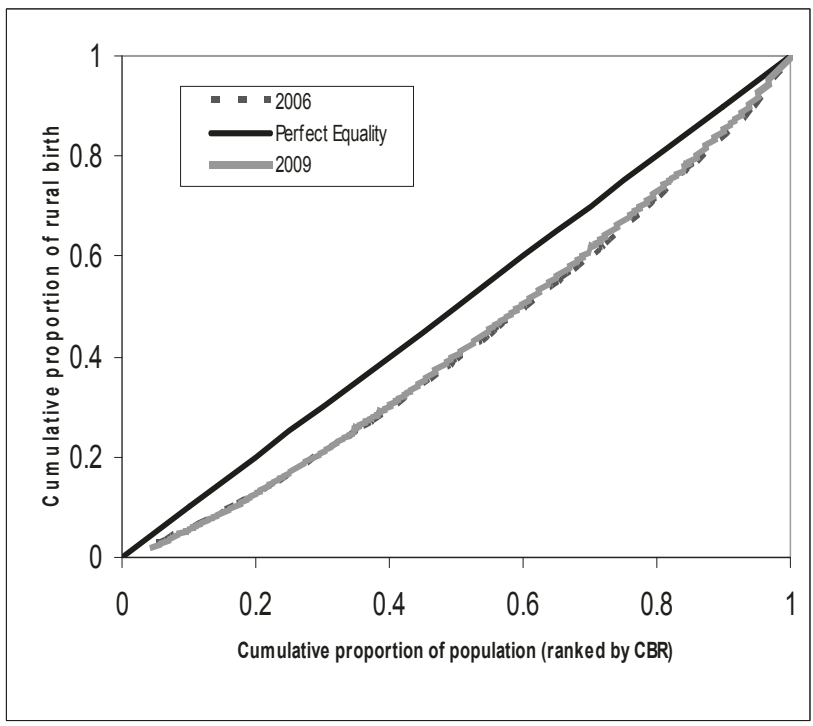

(c)

*Rural health houses

**Crude Mortality Rate (per 1000 rural people)

*** Crude Birth Rate (per 1000 rural people)

Figure 2 Lorenz curves of distribution of: (a) RHHs, (b) CMR and (c) CBR among provinces in Iran 
Table 3 Inequality indicators of the distribution of RHHs, Deaths and Births in rural area of Iran

\begin{tabular}{|c|c|c|c|c|c|c|}
\hline \multirow[t]{3}{*}{ Variable } & \multicolumn{6}{|c|}{ Total sample } \\
\hline & \multicolumn{3}{|c|}{2006} & \multicolumn{3}{|c|}{2009} \\
\hline & Gini coefficient & Index of Dissimilarity & Decile ratio & Gini coefficient & Index of Dissimilarity & Decile ratio \\
\hline$\overline{\mathrm{RHH}}$ & 0.125 & $9 \%(1505)$ & 2.38 & 0.133 & $10 \%(1670)$ & 2.58 \\
\hline Death & 0.260 & $18 \%(27335)$ & 6.70 & 0.206 & $14 \%(18887)$ & 3.70 \\
\hline \multirow[t]{2}{*}{ Birth } & 0.158 & $11 \%(43732)$ & 2.58 & 0.141 & $10 \%(40338)$ & 2.61 \\
\hline & \multicolumn{6}{|c|}{ Outliers excluding sample $^{b}$} \\
\hline $\mathrm{RHH}^{\mathrm{a}}$ & 0.126 & $9 \%(1435)$ & 2.59 & 0.135 & $10 \%(1602)$ & 2.58 \\
\hline Death & 0.185 & $12 \%(15189)$ & 4.75 & 0.177 & $12 \%(14815)$ & 3.19 \\
\hline Birth & 0.162 & $11 \%(42991)$ & 2.96 & 0.143 & $10 \%(38860)$ & 2.70 \\
\hline
\end{tabular}

a Rural health house.

b Three provinces (Hormozgan, Semnan and South Khorasan) were excluded as outliers.

moderate direct statistically significant relationship between CMR and RHP in 2009. On the other hand, the relationship between CBR and RHP was moderately negative in 2006 and 2009; implying that people with higher health needs had lower access to RHHs. When we excluded the provinces with outliers, there were no significant associations between access and need indicators.

\section{Discussion}

The current study assessed access to and need for health services in rural areas of Iran for the years 2006 and 2009. The study showed that the distribution of RHHs is not based on need in terms of CMR and CBR across the provinces in Iran. Moreover, the results indicated significant regional variations in both access and need indicators across the country. To achieve an equal distribution of RHHs across the country, about one out of 10 RHHs should be re-allocated from the relatively over-served provinces to the relatively under-served ones.

The results showed that RHHs are not distributed based on CMR and CBR in the provinces. One possible

Table 4 Correlation between RHP, CMR and CBR in Iran in 2006 and 2009

\begin{tabular}{|c|c|c|}
\hline \multirow[t]{2}{*}{ Variable } & \multicolumn{2}{|c|}{ Total sample } \\
\hline & 2006 & 2009 \\
\hline & $\mathrm{RHP}^{\mathrm{a}}$ & RHP \\
\hline$C M R^{b}$ & $-0.15(0.42)^{d}$ & $0.37(0.04)$ \\
\hline \multirow[t]{2}{*}{$\mathrm{CBR}^{\mathrm{c}}$} & $-0.34(0.06)$ & $-\mathbf{0 . 4 1}(0.03)$ \\
\hline & \multicolumn{2}{|c|}{ Outliers excluding sample ${ }^{e}$} \\
\hline CMR & $-0.06(0.77)$ & $0.31(0.12)$ \\
\hline CBR & $-0.24(0.24)$ & $-0.31(0.11)$ \\
\hline
\end{tabular}

a Rural health houses per 1000 rural people.

b Crude Mortality Rate (per 1000 rural people).

c Crude Birth Rate (per 1000 rural people).

d Spearman rank order correlation with $p$ values in parentheses.

e Three provinces (Hormozgan, Semnan and South Khorasan) were excluded as outliers. explanation for these results is maybe that policy makers consider some other indicators than those used in this study (CMR and CBR) for the distribution of RHHs across the country (for example; having a minimum number of RHHs for each province or socio-economic situation of the provinces).

Although, access to RHHs (measured by RHP) improved between 2006 and 2009, the needs for health services (measured by CMR and CBR) increased at the same time. In terms of inequality measures, there were changes in need indicators toward more equal distributions, while inverse trend was seen for the access indicator.

The degree of inequality in the distribution of $\mathrm{RHH}$ and the CBR were rather stable during the study period, while it significantly changed for CMR in total sample. However, when we excluded the outliers, then no significant changes in inequality indicators were observed. Generally, there was no strong association between the distribution of RHH and CMR and CBR in rural areas of Iran.

Previous studies mostly evaluated the rural-urban differences in distribution of health care resources and outcomes, not differences within the rural areas [14]. Among the few studies within rural areas, Theodorakis et al [23] reported an uneven distribution of primary care physicians in remote areas of Greece and Albania. Another study in the USA in 2005 indicated unequal distribution of physician among rural areas [24].

The results of this study however should be interpreted in light of some limitations. Firstly, the data are gathered from census data which are subject to incompleteness and measurement errors and these may bias the results. For example, undercounting, misreporting and delayed registration are some well known problems of census and mortality data in Iran [25]. These possibly explain the outliners in our data. Secondly, the data used in the study are aggregated data at the province level. It implies that the variation within the provinces could be higher than the variation between the provinces. Hence, these results are not necessarily applicable to smaller geographic units 
such as counties or cities. Thirdly, need variables used in this study were crude measures and sex and age differences in these measures were not taken into account due to the lack of data. Fourthly, our results are limited to geographical comparisons; without knowing who actually use the services provided by RHHs, one cannot know their distribution according to other dimensions of the population, such as income, education and etc.

\section{Conclusion}

This study showed that the distribution of RHHs does not reflect the needs for health care in terms of CMR and CBR. There were significant variations in the distribution of RHHs, CMR and CBR across the country. While the inequality in access increased during the study period, the inequality in need for health care decreased at the same time in the rural areas. It is suggested that the results of this study be considered in making decisions on rural health care services by policymakers in Iran.

\section{Acknowledgements}

It is acknowledged that the current study was supported by the School of Management and Medical Informatics, Tehran University of Medical Sciences.

\section{Author details}

'Division of Health Economics, Department of Clinical Sciences, Malmö; Skåne University Hospital; Lund University, MALMÖ SE-20502, Sweden. ${ }^{2}$ Health Economics \& Management, Institute of Economic Research, Lund University, Lund, Sweden. ${ }^{3}$ Department of Health Management and Economics, School of Public Health, Tehran University of Medical Sciences, Tehran, Iran. ${ }^{4}$ Department of Health Care Management, School of Management and Medical Informatics, Tehran University of Medical Sciences, Tehran, Iran. ${ }^{5}$ School of Public Health, Ardebil University of Medical Sciences, Ardebil, Iran. ${ }^{6}$ Department of Public Health Sciences, Division of Global Health, Karolinska Institute, Stockholm, Sweden.

\section{Authors' contributions}

AAK participated in design of study, analysis and interpretation and drafting the manuscript. BN participated in acquisition the data, acquisition of funding and revision of the manuscript. HHB participated in interpretation of results and revision of the manuscript. All authors read and approved the final manuscript.

\section{Competing interests}

The authors declare that they have no competing interests.

Received: 3 June 2011 Accepted: 22 September 2011

Published: 22 September 2011

\section{References}

1. World Health Organization (1975): Declaration of Alma-Ata. [http://www. who.int/hpr/NPH/docs/declaration_almaata.pdf].

2. The World Health Report: Primary health care, now more than even. Geneva World Health Organization; 2008.

3. Iranian health houses open the door to primary care, WHO Bulletin 86: 8. [http://www.who.int/bulletin/en/]

4. Hanson K, Ranson K, Oliveira-Cruz V, Mills A: Expanding access to priority health interventions: a framework for understanding the constraints to scaling-up. J Int Dev 2003, 15:1-14.

5. Taylor DH, Ricketts TC, Kolimaga JT: Defining under-service and physician shortage areas in historical and future context. North Carolina Rural
Health Research Program, University of North Carolina, Working Paper No.30 1994.

6. CSDH: Closing the gap in a generation: Health equity through action on the social determinants of health. Final Report of the Commission on Social Determinants of Health. Geneva, World Health Organization; 2008.

7. Abasolo I, Manning R, Jones AM: Equity in utilization of and access to public sector GPs in Spain. Applied Economics 2001, 33:349-364.

8. Isfahani DS, Shavazi MJA, Chavoshi MH: Family planning and fertility decline in rural Iran: the impact of rural health clinics. Health Economics 2010, 19:159-180.

9. Aghajanian A, Mehryar AH, Ahmadnia S, Kazemipour S: Impact of rural health development programme in the Islamic Republic of Iran on ruralurban disparities in health indicators. Eastern Mediterranean Health Journal 2007, 13(6):1466-75.

10. Regional Office for the Eastern Mediterranean: Country profile of Iran. 2010 [http://www.emro.who.int/emrinfo/index.aspx].

11. Office for Family Health and Population: Undersecretary for Health. Iran Population Data Report. Tehran, MOHME; 2003.

12. Statistical Centre of Iran. Iran statistical year books. [http://www.amar.org. ir/default.aspx?tabid $=52$.

13. National Organization for Civil Registration. [http://www.sabteahval.ir/en/].

14. Wilkinson D: Inequitable distribution of general practitioners in Australia: analysis by state and territory using census data. Aust J Rural Health 2000, 8:87-93.

15. Gravelle H, Sutton M: Inequality in the geographical distribution of general practitioners in England and Wales 1974-1995. J Health Serv Res Policy 2001, 6:6-13.

16. Gravelle $H$, Sutton $M$ : Trends in geographical inequalities in provision of general practitioners in England and Wales. Lancet 1998, 352:1910.

17. Chang RKR, Halfon N: Geographic Distribution of Pediatricians in the United States: An Analysis of the Fifty States and Washington, DC. Pediatrics 1997, 100:172-179.

18. Theodorakis PN, Mantzavinis GD, Rrumbullaku L, Lionis C, Trell E: Measuring health inequalities in Albania: a focus on the distribution of general practitioners. Human Resources for Health 2006, 4:5.

19. Bidgoli HH, Bogg L, Hasselberg M: Pre-hospital trauma care resources for road traffic injuries in a middle-income country- A province based study on need and access in Iran. Injury 2010.

20. Brown MC: Using Gini-style indices to evaluate the spatial patterns of health practitioners: theoretical considerations and an application based on Alberta data. Soc Sci Med 1994, 38:1243-1256.

21. Regidor E: Measures of health inequalities: part 1. J Epidemiol Community Health 2004, 58:858-61.

22. Buck D, Smith K: The distribution of community psychiatric nurses in England: are they where they should be? Journal of Advanced Nursing 1998, 28(3):508-516.

23. Theodorakis PN, Mantzavinis GD: Inequalities in the distribution of rural primary health care physicians in two remote neighboring prefectures of Greece and Albania. Rural and Remote Health 2005, 5:457.

24. Fordyce MA, Chen FM, Doescher MP, Hart LG: 2005 Physician Supply and Distribution in Rural Areas of the United States. Rural Health Research Center University of Washington; 2007, Report No.116.

25. Khosravi A, Taylor R, Naghavi M, Lopez AD: Mortality in the Islamic Republic of Iran, 1964-2004. Bull World Health Organ 2007, 85(8):607-14.

doi:10.1186/1475-9276-10-39

Cite this article as: Ahmad Kiadaliri et al:: Geographic distribution of need and access to health care in rural population: an ecological study in Iran. International Journal for Equity in Health 2011 10:39. 